University of Wollongong

Research Online

Faculty of Engineering and Information

Faculty of Engineering and Information

Sciences - Papers: Part B

Sciences

2018

Softer is Harder: What Differentiates Soft Robotics from Hard Robotics?

Gursel Alici

University of Wollongong, gursel@uow.edu.au

Follow this and additional works at: https://ro.uow.edu.au/eispapers1

Part of the Engineering Commons, and the Science and Technology Studies Commons

Research Online is the open access institutional repository for the University of Wollongong. For further information contact the UOW Library: research-pubs@uow.edu.au 


\title{
Softer is Harder: What Differentiates Soft Robotics from Hard Robotics?
}

\begin{abstract}
This paper reports on what differentiates the field of soft (i.e. soft-bodied) robotics from the conventional hard (i.e. rigid-bodied) robotics. The main difference centres on seamlessly combining the actuation, sensing, motion transmission and conversion mechanism elements, electronics and power source into a continuum body that ideally holds the properties of morphological computation and programmable compliance (i.e. softness). Another difference is about the materials they are made of. While the hard robots are made of rigid materials such as metals and hard plastics with a bulk elastic modulus of as low as $1 \mathrm{GPa}$, the monolithic soft robots should be fabricated from soft and hard materials or from a strategic combination of them with a maximum elasticity modulus of $1 \mathrm{GPa}$. Soft smart materials with programmable mechanical, electrical and rheological properties, and conformable to additive manufacturing based on 3D printing are essential to realise soft robots. Selecting the actuation concept and its power source, which is the first and most important step in establishing a robot, determines the size, weight, performance of the soft robot, the type of sensors and their location, control algorithm, power requirement and its associated flexible and stretchable electronics. This paper outlines how crucial the soft materials are in realising the actuation concept, which can be inspired from animal and plant movements.

Disciplines

Engineering | Science and Technology Studies

Publication Details

Alici, G. (2018). Softer is Harder: What Differentiates Soft Robotics from Hard Robotics?. MRS Advances, 3 (28), 1557-1568.
\end{abstract}




\title{
Softer is harder: what differentiates soft robotics from hard robotics?
}

\author{
Gursel ALICI \\ School of Mechanical, Materials, Mechatronic and Biomedical Engineering \\ ARC Center of Excellence for Electromaterials Science \\ University of Wollongong, 2522 NSW, Australia
}

Email: gursel@uow.edu.au

\begin{abstract}
This paper reports on what differentiates the field of soft (i.e. soft-bodied) robotics from the conventional hard (i.e. rigid-bodied) robotics. The main difference centres on seamlessly combining the actuation, sensing, motion transmission and conversion mechanism elements, electronics and power source into a continuum body that ideally holds the properties of morphological computation and programmable compliance (i.e. softness). Another difference is about the materials they are made of. While the hard robots are made of rigid materials such as metals and hard plastics with a bulk elastic modulus of as low as $1 \mathrm{GPa}$, the monolithic soft robots should be fabricated from soft and hard materials or from a strategic combination of them with a maximum elasticity modulus of 1 GPa. Soft smart materials with programmable mechanical, electrical and rheological properties, and conformable to additive manufacturing based on 3D printing are essential to realise soft robots. Selecting the actuation concept and its power source, which is the first and most important step in establishing a robot, determines the size, weight, performance of the soft robot, the type of sensors and their location, control algorithm, power requirement and its associated flexible and stretchable electronics. This paper outlines how crucial the soft materials are in realising the actuation concept, which can be inspired from animal and plant movements.
\end{abstract}

\section{INTRODUCTION}

Although there is no commonly agreed definition for robotics, we define robotics as the science and engineering of devices which are reprogrammable, multi-functional, multi-purpose and versatile systems intelligently linking sensing to action. This definition can be extended to soft robotics as the science and engineering of the robots primarily made of soft materials, components and monolithic active structures such that they can safely interact with and adapt to their immediate environment better than the robots made of hard components $[1-4,15]$. Soft robotics, which is sometimes called biologically inspired robotics, offers unprecedented solutions for applications involving smooth touches, safe interaction with humans, manipulating and grasping fragile objects, crops and similar agricultural products. Soft robots can be in the form of robot manipulators, grippers, medical robots, agricultural robots, rehabilitation robots and similar, depending on the application. It must be noted that although the robots can be classified according to their key features such as drive system, degrees of freedom, kinematic structure, work-volume, type of motion etc, they can be classified, at the system level, into walking robots, humanoid robots, wheeled robots (i.e. mobile robots), robot manipulators (i.e. industrial robots) and similar. This paper refers to the robot manipulators as described by ISO 8373:2012 [13] though some of the principles and concepts presented in this paper are general enough to apply to most of robots. 
Robots can be made of a number of rigid links connected to each other with a single degree of freedom rigid or elastic joints, like hyper-redundant manipulators or invertebrate-like robotic topologies, where each joint is controlled independently to realize a task or purpose [5-7]. This is a classical approach which has been used in many robotic designs. However, this approach requires intricate algorithms to control (i) the position of each link and whole robot and/or (ii) the contact force during the physical interaction and interface of the robot with its environment. In line with recent progress in soft smart materials or electromaterials and additive manufacturing techniques, the soft robots consist of a monolithic (i.e. whole) body containing actuation and sensing elements, mechanical structure, energy storage units with a minimum foot-print. Such robots are expected to change their effective stiffness in order to provide a desired force or compliance when interacting with their environments including physical interaction with humans.

When establishing a soft robot, compromise should be made among its weight, footprint, cost, life, dexterity, robustness, and control complexity. This depends on the optimal number and placement of the actuators, sensors, motion transmission elements (i.e, mechanisms), and mechanical compliance, which are decided at the design stage. The fundamental question is how to place actuators and sensors that will require minimum control effort without trading off the safety and performance of the robot.

Another important question is how to integrate the concept of morphological computation into the design of soft robots [8, 9]; the control functions are distributed over the morphology, materials, and constraints associated with the soft robot and its interface with the environment. In simple terms, morphological computation requires outsourcing the computation and decision making to the morphology of the robot or characteristics of the materials it is made of. An under-actuated prosthetic hand grasping an unknown object is a good example to morphological computation [10]. Animal locomotion is based on adapting the stiffness and damping of muscles to the terrain or operation environment, rather than precisely controlling the trajectory of the limbs and joints facilitating the locomotion; the morphology of the muscles is adjusted to the occasion. If the joints of a limb or limbs are individually controlled, a significant amount of dynamic computation will be required. The concept of morphological computation also introduces embodied intelligence into soft robots.

Materials with programmable mechanical, rheological and electrical properties are essential to integrate the morphological computation into the design of soft robotic systems. Soft robots should adjust their effective mechanical compliance to make a safe and adaptive interaction with their environment. They should tune their stiffness to adapt or self-organise to the occasion. Mechanical compliance which distinguishes soft robots from hard robots distributes contact forces evenly on a larger surface with less pressure on contacting surfaces [18]. The conventional robot components are made of the materials with the bulk modulus of elasticity of at least $1000 \mathrm{MPa}$. On the other hand, the living organs such as skin, muscle tissue and cartilage have a modulus of elasticity in the range of $100 \mathrm{~Pa}$ to $1 \mathrm{GPa}[1,16,18]$. This is a significant compliance mismatch (hard robot + soft environment) that can generate high stress concentrations and contact forces potentially causing damage. The compliance matching is especially important for human-machine interactions. When there is an application where a safe human-machine interaction and adaptability with the physical environment is required, there will be a need for robotic systems or components with variable stiffness. It must be noted that the concept of modulus of elasticity is valid to describe the softness or hardness of uniform 
materials tested as a prismatic bar axially loaded with a small strain within the elastic range of the material. In this regard, the softness of the materials for soft robotics cannot be described with this classical concept of modulus of elasticity. However, it is used in the soft robotic literature to compare the relative softness of the soft robots to that of the hard robots.

The progress in soft robotics strongly depends on the progress in the science and technology of smart materials (or active materials or live materials or robotic materials) which are amenable to additive manufacturing or moulding techniques [16, 19]. Soft robots require structural materials as well as responsive or active materials. The mechanical and electrical properties of the active materials play a significant role to make a soft or gentle contact with the physical environment. Rather than using the well-studied and challenging research topic of force control [44] based on intricate control rules and requirements, the programmable or variable properties of the active material will allow to make a soft and smooth interaction with the environment.

The most important step in establishing a soft robot is to determine its energy efficient, low foot-print and low weight actuation principle. The actuation principle determines the size, weight, performance of the soft robot, its sensors and their location, control algorithm, power requirement and its associated flexible and stretchable electronics. Therefore, the primary focus of this paper is to discuss the significance of soft materials in establishing the soft robots and especially their actuation concept. The characteristics of soft and printable actuator materials are described in the light of (i) what features of soft robotic systems differentiate them from the conventional hard robotic systems, and (ii) the compliance of the soft robots should be less than that of the object or environment they are interacting with. We specifically consider soft robots in the form of a monolithic (i.e. continuum) body, much like squid tentacles, elephant trunks and tongues, which can operate either in the bending mode (bending actuators) or the longitudinal mode (linear actuators).

\section{WHAT ARE THE ELEMENTS OF A TYPICAL CONVENTIONAL ROBOTIC SYSTEM AND A SOFT ROBOTIC SYSTEM?}

Soft robots cannot replace the conventional robots. However, for some applications, especially for medical applications, the soft robots are ideal. A comparison of the conventional and soft robotic systems is presented in Table 1 . A schematic comparison of a rigid-bodied robotic arm, a redundant arm, and a corresponding soft robotic arm are presented in Figure 1 to illustrate the fundamental differences between hard robotics and soft robotics.

As illustrated in Figure 2, conventional robotic systems (i.e., rigid-bodied robots) which typically consist of actuators, sensors, motion transmission and conversion mechanism, mechanical structure, and interface with its controller and outside world use hard components and conventional actuators to meet the strict requirements of industrial automation, control, and manufacturing. Of these essential elements, choosing the most efficient actuator is crucial not only for the overall performance of the robot, but also for determining other elements and their configurations. A comparison of the actuators with human muscles is provided in Table 2. Despite many research efforts, there are no actuators which can fully replicate the performance of natural muscles. 
Table 1: Characteristics of soft and hard robotic systems.

\begin{tabular}{|c|c|}
\hline Soft Robotic Systems & Hard Robotic Systems \\
\hline $\begin{array}{l}\text { Made of soft, flexible, stretchable materials } \\
\text { with reversible and variable properties }\end{array}$ & $\begin{array}{l}\text { Made of hard materials with invariable } \\
\text { properties }\end{array}$ \\
\hline $\begin{array}{l}\text { Inherent compliance match with its } \\
\text { environment }\end{array}$ & $\begin{array}{l}\text { Smooth contact with its environment } \\
\text { facilitated by advanced feedback control } \\
\text { strategies and sensors }\end{array}$ \\
\hline $\begin{array}{l}\text { Continuum topology with infinite degrees of } \\
\text { freedom (DoF) seamlessly housing all of its } \\
\text { essential elements }\end{array}$ & $\begin{array}{l}\text { Discrete topology with a finite DoF } \\
\text { consisting of rigid elements connected to } \\
\text { each other with single DoF joints. }\end{array}$ \\
\hline $\begin{array}{l}\text { Inherently safe, adaptive and tolerant to } \\
\text { operate in unknown environments, } \\
\text { especially for human-machine interaction }\end{array}$ & $\begin{array}{l}\text { Unsafe and intolerant with limited } \\
\text { adaptability to operate in unknown } \\
\text { environments unless intricate control } \\
\text { measures are applied. }\end{array}$ \\
\hline $\begin{array}{l}\text { Flexible and stretchable electronics and } \\
\text { power source }\end{array}$ & Conventional electronics and power source \\
\hline vel of behavioural diversity & el of behc \\
\hline High level of bi & Lov \\
\hline uracy & Hig \\
\hline eed and for & peed and for \\
\hline Low weight and cost & High weight and cost \\
\hline
\end{tabular}

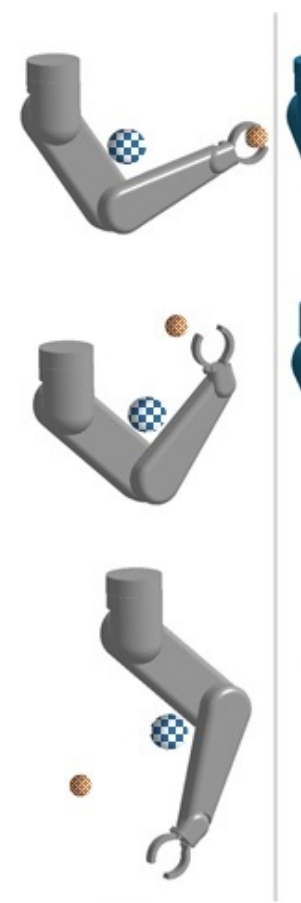

(a)

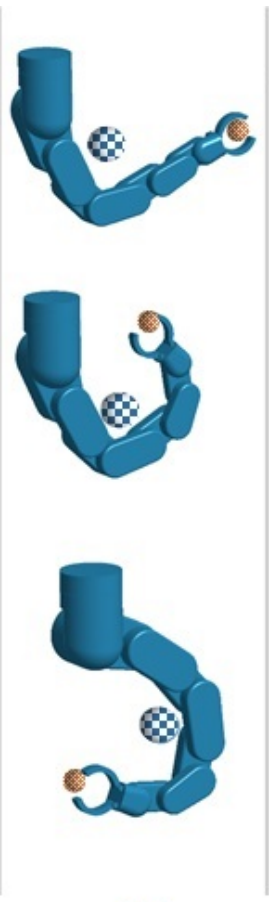

(b)

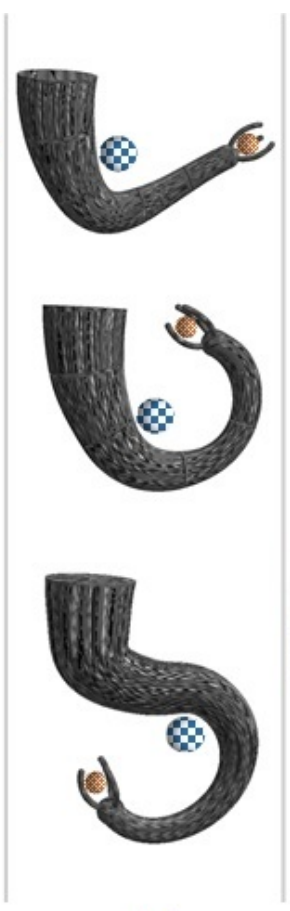

(c)

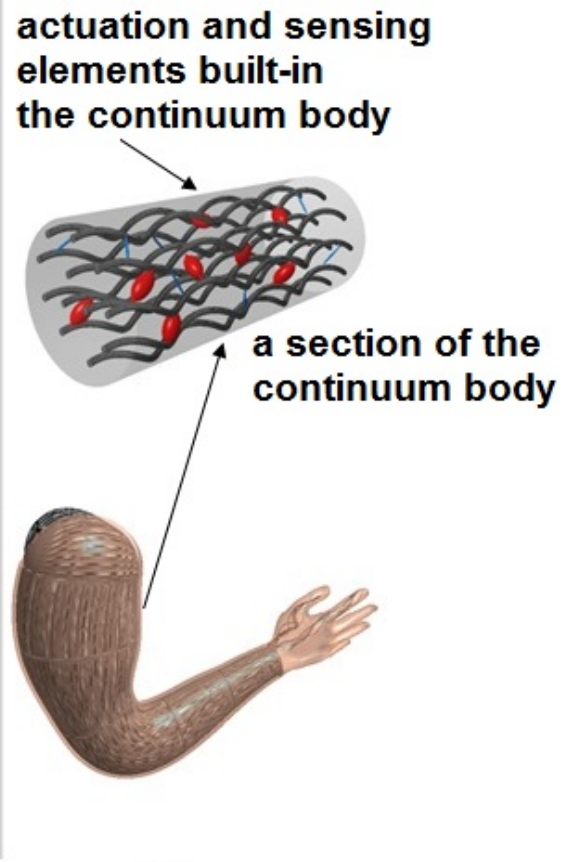

(d)

Figure 1: Comparison of (a) a conventional robotic manipulator, (b) a corresponding hyper-redundant robotic manipulator, (c) a corresponding soft robotic manipulator, and (d) a soft robotic arm with a continuum topology seamlessly housing the actuation and sensing elements in the same continuum body. 


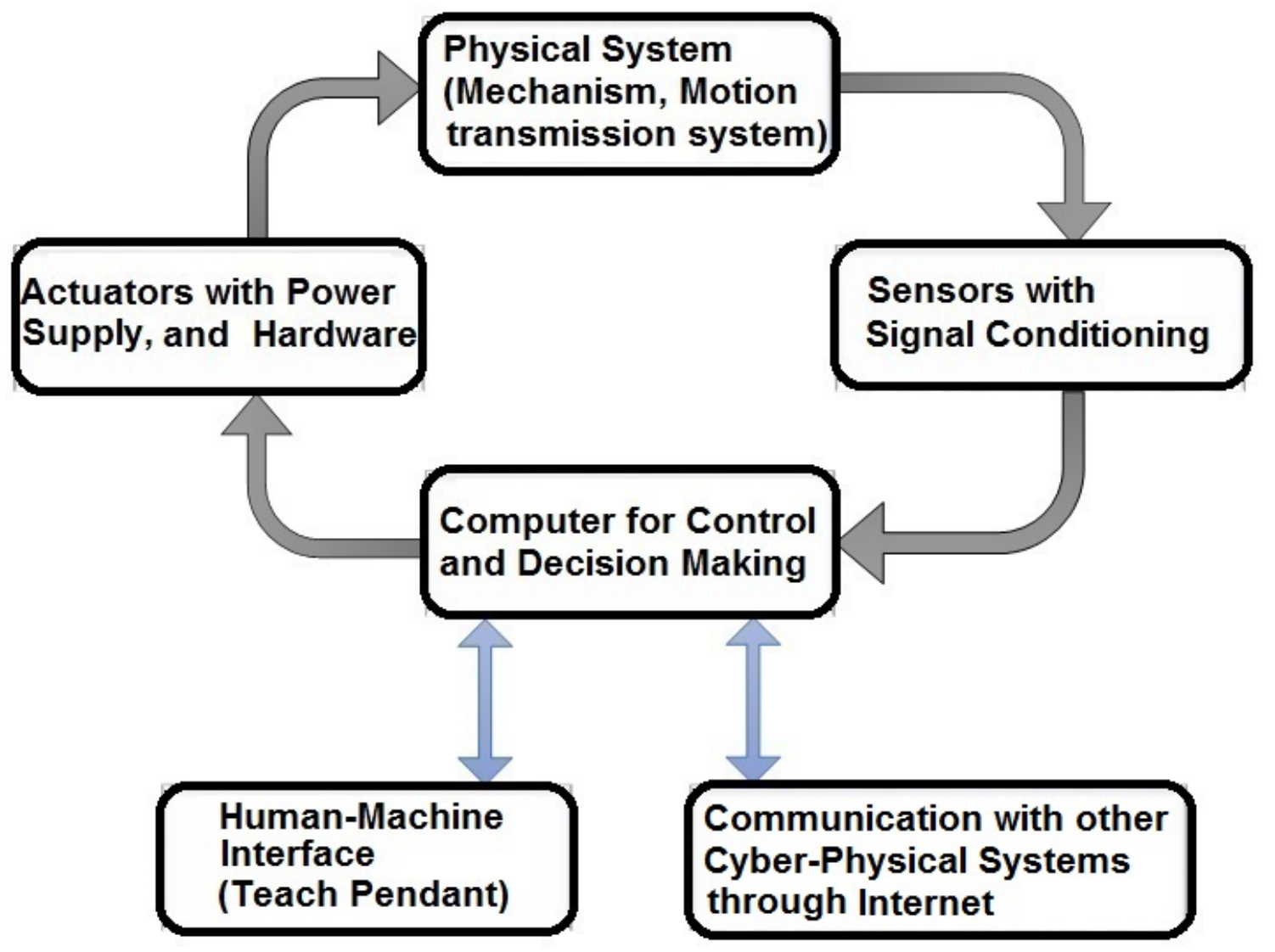

Figure 2: Elements of a typical robotic system.

Table 2. A comparison of typical robotic actuators with the human muscle with approximate values, compiled from a range of sources [11, 35, 39, 42, 45]. Specific power $\boldsymbol{\rho}$ is the power per unit of weight, $\boldsymbol{\sigma}_{\max }$ is the maximum force applied by the actuator per unit area (i.e. maximum actuation stress), $\boldsymbol{\varepsilon}_{\boldsymbol{m a x}}$ is the maximum actuation strain. Maximum stress and maximum strain are valid for linear actuators. E is the actuator modulus of elasticity. Efficiency is the mechanical efficiency. $f_{\max }$ is the maximum operation frequency. The indexes without a numerical value depend on too many operation and design parameters. Therefore, they are left blank. EAP (I) and EAP (N/I) refer to the ionic EAO and non-ionic EAP, respectively.

\begin{tabular}{ccccccc}
\hline Actuator type & $\varepsilon_{\max }$ & $\sigma_{\max }(\mathrm{MPa})$ & $\mathrm{E}(\mathrm{MPa})$ & $\rho[\mathrm{W} / \mathrm{kg}]$ & Efficiency & $f_{\max }(\mathrm{Hz})$ \\
\hline Muscle & $0.3-0.4$ & $0.1-0.4$ & $5-20$ & $50-284$ & $0.2-0.4$ & $50-500$ \\
\hline DC Motors & 0.4 & 0.1 & -- & 100 & $0.6-0.9$ & --- \\
Pneumatic & $0.1-1$ & $0.5-0.9$ & $50-90$ & 4000 & $0.4-0.5$ & $50-300$ \\
Hydraulic & $0.1-1$ & $20-70$ & $2-3 \times 10^{3}$ & $1600-2000$ & $0.9-0.98$ & $50-300$ \\
SMA & 0.07 & $100-700$ & $3-9 \times 10^{4}$ & $6400-6600$ & $0.01-0.02$ & $0.02-0.07$ \\
SMP & 1.0 & $2-14$ & $4-12 \times 10^{3}$ & $850-880$ & $<0.1$ & $<0.01$ \\
EAP (I) & $0.02-0.4$ & $5-34$ & $0.2-3 \times 10^{3}$ & 150 & $<0.01$ & $1-500$ \\
EAP (N/I) & $0.2-3.8$ & $5-6$ & 1 & $1000-2500$ & $0.15-0.9$ & $1-2000$ \\
MREs & 0.005 & $0.1-10$ & $1-10$ & $3-4 \times 10^{3}$ & $0.6-0.8$ & -- \\
MRFs & 0.002 & 0.1 & -- & $3-4 \times 10^{3}$ & -- & -- \\
\hline
\end{tabular}


A typical soft robotic system should be designed to have an integrated topology with the same elements as the conventional hard robotic systems, except all of these features should be built into a monolithic body like live bodies such as animals and plants. Depending on the application, the actuation principle can be inspired from human beings, animals, and their mammalian skeleton muscles or from plants [8,34,36,38,42].

\section{IT IS ALL ABOUT ACTUATION}

Selecting the actuation concept is the first and most important step, which affects the size, weight, performance of the soft robot, the type of sensors and their location, control architecture, power requirement for the aimed soft robot. The progress in soft robotics significantly depends on the progress in actuation concepts [40]. Actuation involves converting a stimulus (i.e. input) into a mechanical output. The stimulus can be in the form of inserting chemical species such as ions (e.g., electroactive polymer actuators), air (pneumatic actuation), liquid (e.g., hydraulic actuation) to cause a volume expansion and /or contraction depending on the actuation topology and configuration or directly applying a stimulus such as heat (e.g., shape memory alloys, shape memory polymers), light (e.g., light responsive polymers), electrical field (e.g., dielectric elastomer actuators), magnetic field (e.g., magnetorheological fluids and elastomers).

We divide the actuators for soft robots into two main classes; extrinsic (not integral part of the robotic system) actuators and intrinsic (built-in) actuators as the integral part of the robotic system or the mechanical structure containing the actuation elements. Pneumatic actuation [20-22, 29-31], hydraulic actuation [23, 27, 33], electric motor driven tendon cable actuation [10], combustion based actuation [24] belong to the extrinsic group. The actuation concepts based on electroactive polymers [25], dielectric elastomers [26], hydrogels [27, 32], shape memory alloys [28], magnetorheological fluids and elastomers [48, 49] and similar belong to the intrinsic group. While the extrinsic actuators are large in size, speed, and mechanical output, the intrinsic actuators are relatively slow with a smaller mechanical output, but have a low footprint. Pneumatic and hydraulic actuation concepts have been the popular choices to demonstrate some proof of concepts for soft actuators and soft robots.

Of the extrinsic actuator, the soft pneumatic actuators which are based on a volumetric expansion or contraction can be designed strategically to generate the required motion pattern though they require a bulky power supply, pumps and valves. One of the most popular pneumatic flexible actuators known as McKibben actuators are not only low weight, low cost, compact, inherently compliant, and damage-tolerant, but also deliver a high power to weight ratio with a high operation life [14]. They are especially suitable for soft robotic applications where compliance matching is required when interaction with a physical environment is essential. One of their drawbacks is their nonlinearity based on length versus pressure and force versus pressure hysteresis, which make their control challenging [12]. In general, soft pneumatic actuators offer the advantages of soft contact with a better conformability, lightweight, high power density, low cost, and easy to manufacture using additive manufacturing and moulding techniques.

Of the intrinsic actuators, electroactive polymer actuators, also known as artificial muscles, require as low as $1.0 \mathrm{~V}$ electrical potential and have a high force output to weight ratio [46, 47]. The dielectric elastomer actuators require high operating voltages higher than $500 \mathrm{~V}$. Hydrogels [32], as soft actuator materials, that change their shape and volume under different 
hydration, $\mathrm{pH}$, light, ionic strength, and temperature conditions generate movement patterns resembling the hydromorphic movement of plants. However, showing a high response time and requiring an aqueous environment for actuation are their major disadvantages if they are used for actuation. Shape memory alloys which are also potential candidates for soft actuators replacing tendon or cable driven type actuation eliminate the use of an external motor. However, their efficiency is quite low due to energy loss to heating, and dissipating the heat gained is a challenge that is directly related to their reversibility [28]. Recently, a composite soft material made of a silicone elastomer matrix containing micro-bubbles filled with ethanol has been proposed for intrinsic soft actuators [41]. This electrically activated composite material requires Joule heating during which ethanol boils at a temperature of $78.4{ }^{\circ} \mathrm{C}$ and subsequently, the pressure in the bubbles increases to expand the elastic silicone matrix in order to generate strains as high as $900 \%$. Though it is a low cost, low density, high strain and high stress material straight forward to synthesise, the actuators made of this material has a quite low efficiency, $\sim 0.2 \%$ and a high response time.

\section{WHAT ARE THE CHARACTERISTICS OF SYNTHETIC MATERIALS FOR SOFT ACTUATORS?}

By a soft actuator we here refer to an articulation mean built in a monolithic (i.e. continuum) body like squid tentacles, elephant trunks and tongues, which do not have an explicit skeletal structure. These belong to the intrinsic class of soft actuators. These appendages, known as muscular hydrostats, are articulated by the expansion and contraction of their constant volume muscles [37]. The monolithic body with a built-in actuator or actuator makes a continuum manipulator with an infinite number of DOFs and configurations such that the manipulator can change or program its shape or configuration and DOFs as per function requirements, like shown in Figure 1c and 1d. We consider two common actuation modes of bending mode and longitudinal mode, which are simultaneously realised in a live continuum body like squid tentacles, elephant trunks and tongues. However, the continuum body of an engineered structure is usually built such that either bending actuation or longitudinal actuation is realised separately. To establish a continuum body manipulator operating either in the bending mode or in the longitudinal mode, the synthetic materials for its actuators should have the following properties;

> Soft; flexible and stretchable, responsive or active, with variable properties (for comparison purpose, e.g., $100 \mathrm{~Pa}<\mathrm{E}$ : tangent modulus $<1000 \mathrm{MPa}$, and $10 \%<$ strain $<100 \%,[1,39,43])$. Tangent modulus which is described for materials deformed beyond their elastic limits under a static load or stress is obtained by taking the slope of their stressstrain plot at a specific point after the elastic region, where the stress-strain relationship is nonlinear. Most of the soft synthetic materials (hydrogels, silicones, polyurethanes, electroactive polymers) available fall within these ranges, especially of the tangent modulus. In principle, the compliance of the soft robot should be less than that of the object or environment it is interacting with. It is reasonable to assume that the monolithic soft robot will interact with humans and similar live and soft objects. Therefore, the modulus range is determined with reference to the mechanical properties of biological materials ranging from body fat to tendon [1]. With reference to high strain required, soft robotic systems will primarily be made of viscoelastic materials. It is, therefore, logical to consider the storage modulus (which is obtained in the frequency domain, under sinusoidal loads or stresses) of the actuator materials, rather than their 
tangent modulus while specifying the material properties. When this point is considered, the maximum limit of the storage modulus of elasticity for synthetic soft materials for soft actuators will be below $1000 \mathrm{MPa}$.

$>$ Inexpensive, amenable to a scalable fabrication method based on additive manufacturing especially 3D printing. If possible, no moulding and post-processing operations should be required,

$>$ Programmable mechanical (e.g., tunable stiffness and damping constants), electrical (e.g, resistance, capacitance) and rheological (e.g., tunable viscoelastic moduli, stress relaxation modulus, shear viscosity) properties for actuation, sensing and printing, respectively.

$>$ Resistant to fatigue under large and reversible strains, and biocompatible (no toxicity or harmful byproducts),

$>$ Integrative and distributive like natural muscles; providing structure, support and actuation seamlessly, (30-80\% of the muscle fibers should contribute to the mechanical output, [42]). It is essential to have a high percentage of the actuator mass to contribute to the force generation.

$>$ Suitable to establishing an efficient sensing principle, flexible and stretchable electronics and power source with an overall low footprint.

$>$ Reversible and predictable behaviour with a reasonably short response time $(<1$ second) under a low foot-print stimulus such as an electrical input.

$>$ An approximately linear response with high sensitivity and negligibly small hysteresis and creep

The field of materials science and synthesis is well developed to follow the "top-to-down approach" in order to formulate and develop soft and functional materials which can meet strict application and function requirements $[16,17]$. Robotics community need soft materials specifically synthesised to meet the function requirements of a soft robot. Especially materials which can directly respond to an electrical potential (i.e. electrically responsive materials or electromaterials) will be ideal to establish an actuation concept. In this regard, the electroactive polymers have been considered as promising materials for soft actuators. However, there are many scientific and technological research questions around their fabrication, packaging, performance and control which must be addressed before they can used to establish a functional actuator or a soft robot. Based on the same reason, materials (e.g., shape memory allow polymers, low melting point alloys etc) which can change their softness (i.e. electrically tuneable softness, [34]) as per electrical input applied to them will be needed to establish structures and topologies with variable stiffness. Electrically activated materials are faster than chemically activated, light-activated, thermally activated, and magnetically activated materials. This requires a soft, flexible, stretchable, high energy density and low foot-print electrical energy source. Though there has been significant progress in battery technologies, they are not yet suitable to integrating them into the monolithic body of a simple robotic topology such as a slender body mimicking a finger of a prosthetic hand.

As much as understanding biomechanics of animals and live creatures, it is important to have materials with properties similar to the properties of biological materials. Therefore, the dream soft materials should have up to $100 \%$ strain. Ideally, soft robotics need actuators with the performance closer to the performance of natural muscles [38]. Depending on the occasion, natural muscles behave like springs with variable stiffness, dampers absorbing energy during 
movement, actuators providing propulsion during movement, transmission elements or mechanisms to transmit forces. This multi-functionality is the property, which should be achieved by varying the stimulus applied to the continuum morphology, preferably an electrical input. The DEA actuators show performance metrics closer or better than that of the mammalian skeletal muscles. However, this can be misleading due to the fact that the actuator topologies based on the DEA cannot provide multi-functionality, self-repair, high cycle life with built-in actuation, sensing, energy source, and heat/waste recovery provided by the natural muscles.

It is important to operate a soft robot well below the maximum or ultimate strain of the material it is made of. When a soft robotic element is loaded closer to its ultimate strain, its operation life will be adversely affected, showing less resistance to fatigue and failure. Therefore, it will be reasonable to keep its deformation or stroke or strain below $70 \%$ of its maximum strain. This operation regime has been essential for artificial muscles to extend their operation life [17, 39].

\section{CONCLUSIONS}

We have presented the main differences between hard robots and soft robots. Soft robots require active materials amenable to $3 \mathrm{D}$ printing such that all essential elements of the robot can be integrated into a monolithic body, with no differentiation among its essential elements of actuators, sensors, mechanical structure, motion transmission and conversion mechanism, electrical elements and power source. The progress in soft robotics strongly depends on the progress in the science and technology of smart materials, which are amenable to additive manufacturing or moulding techniques. Robotics community need materials suitable to fabricating energy efficient adaptive and integrative topologies containing the essential elements. The progress in soft materials with predictable behaviour should be matched by the progress in additive manufacturing. It is a significant challenge to print soft materials or elastomeric materials with viscoelastic and nonlinear properties. Of course, other additive manufacturing techniques such as moulding can be used until the technology is ready for 3D printing more complex structures and robots with soft smart materials.

\section{ACKNOWLEDGMENTS}

This study is supported by ARC Centre of Excellence for Electromaterials (ACES) (Grant No. CE140100012), and the Intelligent Nano-Tera Research Systems Laboratory at University of Wollongong. The author thanks Professor Gordon G Wallace for his suggestion for the title of this paper, and Dr Rahim Mutlu for his help in preparing Figure 1.

\section{REFERENCES}

1. D. Rus and M. T. Tolley, Nature, 521, no. 7553, 467-475, (2015).

2. S. Kim, C. Laschi, and B. Trimmer, Trends in Biotechnology, 31:5, 287-294, (2013).

3. S. Bauer, S. Bauer-Gogonea, I. Graz, M. Kaltenbrunner, C. Keplinger, and R. Schwoediauer, Adv. Mater. 26, 149-162, (2014). 
4. G. Alici, Editorial for Special Issue, http://www.mdpi.com/journal/robotics/special_issues/soft_robotic, accessed on May 20, 2016.

5. G. S. Chirikjian and J. W. Burdick, IEEE Transactions on Robotics and Automation, 11:6, 781 - 793, (2005).

6. R. Kang, D. T. Branson, T. Zheng, E. Guglielmino, and D. G. Caldwell, Bioinspiration \& biomimetics, 8:3, p. 036008, (2013).

7. R. J. Webster and B. A. Jones, The International Journal of Robotics Research, 29:13, 1661-1683, (2010).

8. R. Pfeifer, M. Lungarella, and F. Iida, Communications of ACM, 55:11, 76 - 87, (2012).

9. H. Hauser, A. J. Ijspeert, R. M. Fuchslin, R. Pfeifer, and W. Mass, Biological Cybernetics, 105, 355 -370, (2011).

10. R. Mutlu, G. Alici, M. in het Panhuis, and G.M, Spinks, Soft Robotics, 3:3, 120 -133, (2016).

11. Huber, J. E., N. A. Fleck and M. F. Ashby, The Royal Society: 2185, (1997).

12. T. V. Minh, T. Tjahjowidodo, H. Ramon, and H. V. Brussel, IEEE/ASME Transactions on Mechatronics, 16:1, 177-186, (2011).

13. ISO 8373:2012, Robots and robotic devices - Vocabulary, https://www.iso.org/obp/ui/\#iso:std:iso:8373:ed-2:v1:en, accessed on June 7, 2017.

14. C. D. Onal, and D. Rus, Bioinspiration \& Biomimetics, 8:2, 026003, 2013.

15. A. Albu-Schaffer, O. Eiberger, M. Grebenstein, S.Haddadin, C. Ott, T. Wimbock, S. Wolf and G. Hirzinger, IEEE Robot. Autom. Mag. 15, 20-30, (2008).

16. R. H. Ewoldt, Soft Robotics, 12-20, (2013).

17. G. Alici, R. Mutlu, D.Melling, E. W. H. Jager and K.Kaneto, In Electromechanically Active Polymers: A Concise Reference, edited by F. Carpi, Springer International Publishing, (2016).

18. C. Majidi, Soft Robotics, 5-11, (2013).

19. M. A. McEvoy and N. Correll, Science 347, DOI: 10.1126/science.1261689, (2015).

20. K. C. Galloway, K. P. Becker, B. Phillips, J. Kirby, S. Licht, D. Tchernov, R. J. Wood, and D. F. Gruber, Soft Robotics, 3, 23-33, (2016).

21. B. Mosadegh, P. Polygerinos, C. Keplinger, S. Wennstedt, R. F. Shepherd, U. Gupta, J. Shim, K. Bertoldi, C. J. Walsh and G. M. Whitesides, Advanced Functional Materials, 24, 2163-2170, (2014).

22. K. Suzumori, S. Iikura, and H. Tanaka, Robotics and Automation, Proceedings., 1991 IEEE International Conference on, 1622-1627, (1991)

23. S. Wakimoto, K. Suzumori, and K. Ogura, Advanced Robotics 25, 1311-1330, (2011).

24. R. F. Shepherd, A. A. Stokes, J. Freake, J. Barber, P. W. Snyder, A. D. Mazzeo, L. Cademartiri, S. A. Morin, and G, M. Whitesides, Angewandte Chemie International Edition, 52, 2892-2896, (2013).

25. R. Mutlu, G. Alici, and W. Li, IEEE/ASME Transactions on Mechatronics, 21, 14671478, (2016).

26. C. H. Nguyen, G. Alici, and R. Mutlu, ASME Journal of Mechanical Design, 136, 061009-061009-9, (2014).

27. H. Yuk, S. Lin, C. Ma, M. Takaffoli, N. X. Fang, and X. Zhao, Nature Communications, 8, 14230, 02/01/online. (2017).

28. H. Jin, E. Dong, G. Alici, S. Mao, X. Min, C. Liu, K. H. Low, and J. Yang, Bioinspiration \& Biomimetics,11, 056012, (2016).

29. H.K. Yap, H.Y. Ng, C.-H. Yeow, Soft Robotics 3(3), 144-158, (2016). 
30. G. Agarwal, N. Besuchet, B. Audergon and J. Paik, Sci. Rep. 6, 34224; doi: 10.1038/srep34224 (2016).

31. D. Yang, B. Mosadegh , A. Ainla , B. Lee , F. Khashai , Z. Suo , K. Bertoldi , and G. M. Whitesides, Advanced Materials, 27, 6323-6327, (2015).

32. S. E. Bakarich, R. Gorkin, M. in het Panhuis and G. M. Spinks, Macromol Rapid Commun, 36:1211-1217, (2015).

33. R. K, Katzschmann, A. D. Marchese, and D. Rus, In Proc. International Symposium on Experimental Robotics, (2014).

34. G. Sumbre, G. Fiorito, T. Flash, and B. Hochner, Curr. Biol. 16, 767-772, 2006.

35. C Zhang and C Rossi, Bioinspir. Biomim. 12 025005, (2017).

36. R. J. Full, In The Handbook of Comparative Physiology, W Dantzler, ed., 853-930, Oxford University Press, Oxford (1997).

37. W. M. Kier and K. K. Smith, Zoological Journal of the Linnean Society, 83, 307-324, (1985).

38. M. H. Dickinson, C. T. Farley, R. J. Full, M.A. R. Koehl, R. Cram and S. Lehman, Science, 288, I 00-106 (2000).

39. Madden, J. D. W., Vandesteeg, N. A., Anquetil, P., Madden, P. G., Takshi, A., Pytel, R. Z., Lafontaine, S. R., Wieringa, P., and Hunter, I. W., IEEE J. Oceanic Eng, 29 (3), 7006-28 (2004)

40. B. Trimmer, Soft Robotics. 4:1, 1-2. https://doi.org/10.1089/soro.2017.29011.bat, (2017)

41. A, Miriyev, K. Stack, K. and H. Lipson, Nat. Commun. 1-8, doi:10.1038/s41467-01700685-3, (2017).

42. Meijer, K., Y. Bar-Cohen, and R. J. Full, SPIE Press, 25-46, (2003).

43. P. Polygerinos, N. Correll, S. A. Morin, B. Mosadegh, C. D. Onal, K. Petersen, M. Cianchetti, M. T. Tolley and R. F. Shepherd, Advanced Engineering Materials, DOI: 10.1002/adem.201700016, (2017).

44. G. Alici and R. W. Daniel, International Journal of Robotics and Automation, 11:2, 62-73, (1996).

45. F.L. Liu, G. Alici, B. Zhang, S. Beirne, and W. Li, Smart Materials and Structures, 24, 035015, (2015).

46. G. Alici, and N. N. Huynh, IEEE/ASME Transactions on Mechatronics, 12, 1, 73 -- 84, (2007).

47. B. Gaihre, G. Alici, G. M. Spinks, and J. M. Cairney, IEEE/ASME Journal of Microelectromechanical Systems, 21:3, 574 - 585, (2012).

48. W. H. Li, Y. Zhou, and T. F. Tian, Rheol Acta, 49, 733-740, DOI 10.1007/s00397-0100446-9, (2010).

49. W.H. Li, H. Du and N.Q. Guo, Materials Science and Engineering A, 371, 9-15, (2004). 